\title{
Article
}

\section{Black Hole Growth in Disk Galaxies Mediated by the Secular Evolution of Short Bars}

Du, M, Debattista, Victor P, Shen, J, Ho, L.C and Erwin, P Available at http://clok.uclan.ac.uk/19649/

Du, M, Debattista, Victor P ORCID: 0000-0001-7902-0116, Shen, J, Ho, L.C and Erwin, P (2017) Black Hole Growth in Disk Galaxies Mediated by the Secular Evolution of Short Bars. Astrophysical Journal Letters, 844 (2). ISSN 2041-8205

It is advisable to refer to the publisher's version if you intend to cite from the work. http://dx.doi.org/10.3847/2041-8213/aa7ecb

For more information about UCLan's research in this area go to http://www.uclan.ac.uk/researchgroups/ and search for < name of research Group>.

For information about Research generally at UCLan please go to http://www.uclan.ac.uk/research/

All outputs in CLoK are protected by Intellectual Property Rights law, including Copyright law. Copyright, IPR and Moral Rights for the works on this site are retained by the individual authors and/or other copyright owners. Terms and conditions for use of this material are defined in the policies page.

\section{CLoK}

Central Lancashire online Knowledge www.clok.uclan.ac.uk

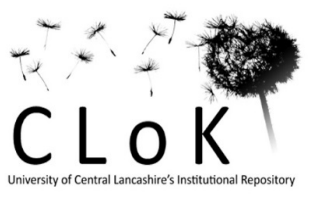




\title{
Black Hole Growth in Disk Galaxies Mediated by the Secular Evolution of Short Bars
}

\author{
Min $\mathrm{Du}^{1,2}$, Victor P. Debattista ${ }^{3,4}$, Juntai Shen ${ }^{1,2}$, Luis C. Ho ${ }^{5,6}$, and Peter Erwin ${ }^{7,8}$ \\ ${ }^{1}$ Key Laboratory of Research in Galaxies and Cosmology, Shanghai Astronomical Observatory, Chinese Academy of Sciences, \\ 80 Nandan Road, Shanghai 200030, China; dumin@ shao.ac.cn \\ ${ }^{2}$ College of Astronomy and Space Sciences, University of Chinese Academy of Sciences, 19A Yuquan Road, Beijing 100049, China; jshen@ shao.ac.cn \\ 3 Jeremiah Horrocks Institute, University of Central Lancashire, Preston PR1 2HE, UK; VPDebattista@uclan.ac.uk \\ ${ }^{4}$ Center for Theoretical Astrophysics and Cosmology, Institute for Computational Science, University of Zürich, \\ Winterthurerstrasse 190, CH-8057 Zürich, Switzerland \\ ${ }^{5}$ Kavli Institute for Astronomy and Astrophysics, Peking University, Beijing 100871, China \\ ${ }^{6}$ Department of Astronomy, School of Physics, Peking University, Beijing 100871, China \\ ${ }^{7}$ Max-Planck-Insitut für extraterrestrische Physik, Giessenbachstrasse, D-85748 Garching, Germany \\ ${ }^{8}$ Universitäts-Sternwarte München, Scheinerstrasse 1, D-81679 München, Germany \\ Received 2017 March 26; revised 2017 June 27; accepted 2017 July 8; published 2017 July 25
}

\begin{abstract}
The growth of black holes (BHs) in disk galaxies lacking classical bulges, which implies an absence of significant mergers, appears to be driven by secular processes. Short bars of sub-kiloparsec radius have been hypothesized to be an important mechanism for driving gas inflows to small scale, feeding central BHs. In order to quantify the maximum BH mass allowed by this mechanism, we examine the robustness of short bars to the dynamical influence of BHs. Large-scale bars are expected to be robust, long-lived structures; extremely massive BHs, which are rare, are needed to completely destroy such bars. However, we find that short bars, which are generally embedded in largescale outer bars, can be destroyed quickly when BHs of mass $M_{\mathrm{bh}} \sim 0.05 \%-0.2 \%$ of the total stellar mass $\left(M_{\star}\right)$ are present. In agreement with this prediction, all galaxies observed to host short bars have BHs with a mass fraction less than $0.2 \% M_{\star}$. Thus, the dissolution of short inner bars is possible, perhaps even frequent, in the universe. An important implication of this result is that inner-bar-driven gas inflows may be terminated when BHs grow to $\sim 0.1 \% M_{\star}$. We predict that $0.2 \% M_{\star}$ is the maximum mass of BHs allowed if they are fed predominately via inner bars. This value matches well the maximum ratio of BH-to-host-galaxy stellar mass observed in galaxies with pseudo-bulges and most nearby active galactic nucleus host galaxies. This hypothesis provides a novel explanation for the lower $M_{\mathrm{bh}} / M_{\star}$ in galaxies that have avoided significant mergers compared with galaxies with classical bulges.
\end{abstract}

Key words: black hole physics - galaxies: evolution - galaxies: kinematics and dynamics - galaxies: nuclei galaxies: structure

\section{Introduction}

It is well established that ellipticals and classical bulges follow tight scaling relations with the masses of their black holes (BHs; see the review by Kormendy \& Ho 2013). Classical bulges are known as remnants of galaxy mergers (e.g., Toomre 1977); these scaling relations led to the prevalence of models involving merger-driven coevolution of BHs and host galaxies (e.g., Di Matteo et al. 2005; Croton et al. 2006). However, bulgeless galaxies and galaxies with pseudobulges, which have a formation history free of significant mergers, do not follow the same scaling relations as classical bulges and ellipticals (Kormendy \& Ho 2013). Many such disk galaxies hosting supermassive BHs have been found (e.g., Filippenko \& Ho 2003; Greene et al. 2010; Jiang et al. 2011; Dong et al. 2012; Jiang et al. 2013; Simmons et al. 2013; Greene et al. 2016), suggesting that significant BH growth can be driven largely by internal secular processes. Deep observations of the galaxy morphology have showed that $\mathrm{BH}$ growth is unlikely to have been driven by significant mergers, at least since redshift $z \sim 2$ (Gabor et al. 2009; Georgakakis et al. 2009; Cisternas et al. 2011; Schawinski et al. 2011; Kocevski et al. 2012; Fan et al. 2014).

Even though bars are considered the most important drivers of secular evolution, how they affect the scaling relations is still not well understood. Near-infrared surveys show that in the nearby universe about two-thirds of disk galaxies host stellar bars (e.g., Eskridge et al. 2000; Menéndez-Delmestre et al. 2007). Almost one-third of barred galaxies also host a short inner bar of general radius $\lesssim 1 \mathrm{kpc}$ (Erwin \& Sparke 2002; Laine et al. 2002; Erwin 2004, 2011), such systems are termed double-barred (S2B) galaxies. At sub-kiloparsec scales, short inner bars have been hypothesized to be an important mechanism for driving gas inflows into the center, efficiently feeding BHs (e.g., Shlosman et al. 1989; Hopkins \& Quataert 2010). Thus, in order to understand the secular growth of BHs, a crucial question is under what conditions short inner bars can survive the presence of a $\mathrm{BH}$.

In triaxial systems, central massive concentrations (CMCs), e.g., BHs, dense stellar clusters, and nuclear disks, can scatter stars moving on elongated orbits onto more chaotic ones (e.g., Gerhard \& Binney 1985). The dissolution of bars under the dynamical influence of BHs has been studied via self-consistent $\mathrm{N}$-body simulations (e.g., Shen \& Sellwood 2004; Athanassoula et al. 2005). Shen \& Sellwood (2004) showed that extremely massive BHs (i.e., more than $4 \%$ of the galaxy stellar mass $M_{\star}$ ) are necessary to destroy large-scale bars. Measurements of $\mathrm{BH}$ masses find a mass range $10^{6-10} M_{\odot}$, and the typical mass ratio of BH-to-host-galaxy stellar mass is $0.1 \%$. Thus, the $\mathrm{BH}$ mass fraction required to destroy bars is rare. Therefore, whether the dissolution of bars has ever happened in the universe has been seriously questioned.

As suggested in Hozumi (2012), shorter bars might be more fragile than normal bars of radius $a_{\mathrm{Bar}}=2-4 \mathrm{kpc}$. In this Letter, we constrain how massive a $\mathrm{BH}$ can become before 
destroying inner bars by adding BHs in the S2B models of Du et al. (2015). In Section 2, we describe the simulations we use. The destruction of short inner bars by BHs is presented in Section 3. A novel explanation of the observed limit in the mass ratio of $\mathrm{BH}$-to-host galaxy is presented in Section 4. We summarize our conclusions in Section 5.

\section{Model Settings}

All the collisionless models we study involve isolated, initially pure-exponential disks. The simulations are evolved with the 3D cylindrical polar option of the GALAXY $N$-body code (Sellwood 2014). The system of units is set to $G=M_{0}=h_{R}=1$, where $G, M_{0}$, and $h_{R}$ are the gravitational constant, mass unit, and scale length of the initial disk, respectively. Physical quantities can be obtained by choosing appropriate scalings. A reasonable scaling to typical spiral/S0 galaxies is $M_{0}=4.0 \times 10^{10} M_{\odot}$ and $h_{R}=2.5 \mathrm{kpc}$, which gives the unit of time $t_{0}=\sqrt{h_{R}^{3} / G M_{0}} \simeq 9.3 \mathrm{Myr}$. We use grids measuring $N_{R} \times N_{\phi} \times N_{z}=58 \times 64 \times 375$, which give rise to a force resolution of 0.01 in the central regions. The disk consists of four million equal-mass particles with softening radius $0.01=25 \mathrm{pc}$. As the central dynamics are largely dominated by the stellar component, we use the same logarithmic rigid halo that $\mathrm{Du}$ et al. (2015) used to simplify the simulations.

In this Letter, we examine the robustness of the well-studied short inner bar of the standard S2B model in Du et al. $(2015,2016)$, which has a disk mass $M_{\star}=1.5 M_{0}$. The initial Toomre- $Q$ is set to $\sim 2.0$ in the outer region; in the inner region, Toomre- $Q$ is reduced gradually toward the center reaching a minimum value of 0.5 . Thus, the inner bar forms spontaneously from the strong bar instability of such a dynamically cool, rotation-dominated, inner disk within a few hundred Myr. At steady state, the semimajor axis of the inner bar is $a_{\text {in }} \sim 0.3 \sim 0.75 \mathrm{kpc}$. The outer bar, which extends to $a_{\text {out }} \sim 3.0 \sim 7.5 \mathrm{kpc}$, forms slowly in the hotter outer disk. In this standard S2B model, the inner bar rotates about three times faster than its outer counterpart.

We also examined the clumpy S2B model whose inner disk fragments, forming clumps at the beginning because of using an even colder inner disk (minimum Toomre- $Q \sim 0.3$ ) than the standard S2B. The clumps move toward the center quickly, then coalesce into an inner bar (Figure 7 in Du et al. 2015) that is relatively stronger, i.e., more massive and longer $\left(a_{\text {in }} \sim 1.5 \mathrm{kpc}\right)$.

The CMC is introduced as the potential of a rigid Plummer sphere

$$
\Phi_{\mathrm{C}}(r)=-\frac{G M_{\mathrm{C}}(t)}{\sqrt{r^{2}+\epsilon_{\mathrm{C}}^{2}}},
$$

where $M_{\mathrm{C}}(t)$ and $\epsilon_{\mathrm{C}}$ are the mass and softening radius, respectively, of the $\mathrm{CMC}$. As $\epsilon_{\mathrm{C}}$ determines the compactness of the $\mathrm{CMC}$, we use $\epsilon_{\mathrm{C}}=0.001=2.5 \mathrm{pc}$ to mimic a BH. The force from the $\mathrm{CMC}$ is added directly to each particle from the analytic form, and is therefore independent of the grid resolution.

In order to mimic the secular growth of $\mathrm{BHs}$, the $\mathrm{BH}$ mass is gradually increased after the bars have reached a steady state at $t_{\mathrm{C}}=300$. The initial CMC mass $M_{\mathrm{C} 0}=0.0001 \% M_{\star}$ grows smoothly to the maximum mass $M_{\mathrm{Cm}}$ in $t_{\mathrm{g}}=50$ time units as follows:

$$
M_{\mathrm{C}}(t)= \begin{cases}M_{\mathrm{C} 0} & \tau<0 \\ \left(M_{\mathrm{Cm}}-M_{\mathrm{C} 0}\right) \sin ^{2}(\pi \tau / 2)+M_{\mathrm{C} 0} & 0 \leqslant \tau \leqslant 1 \\ M_{\mathrm{Cm}} & \tau>1,\end{cases}
$$

where $\tau=\left(t-t_{\mathrm{C}}\right) / t_{\mathrm{g}}$. The growth time $t_{\mathrm{g}}$ is much longer than the dynamical timescale of the central particles. Thus, it can be regarded as an adiabatic growth. The simulations last 800 time units, $\simeq 7.4 \mathrm{Gyr}$ in our standard scaling.

The maximum mass of the $\mathrm{BH}, M_{\mathrm{bh}}$, is varied in the range of $0.01 \% M_{\star}$ to $0.2 \% M_{\star}$. To ensure accurate integration for rapidly moving particles, we reduce time steps by half for adding guard shells around the BH (Shen \& Sellwood 2004). The time step is $\Delta t=0.01$ outside of the guard shells. We introduce eight shells at $r \leqslant 0.12$, the shortest time step reaches $\Delta t / 2^{8}$, which is sufficiently small even for the fastest moving particles.

\section{The Dissolution of Short Inner Bars}

\subsection{The Standard S2B Model}

As shown in Figure 1, the inner bar is completely destroyed within 100 time units by a $\mathrm{BH}$ of mass $M_{\mathrm{bh}}=0.2 \% M_{\star} \sim$ $10^{8} M_{\odot}$. Figure 2 shows the evolution of the inner $\left(A_{\text {in }}\right)$ and outer $\left(A_{\text {out }}\right)$ bar amplitudes. The black profiles represent the model using a tiny $\mathrm{BH}$ of fixed mass $0.0001 \% M_{\star}$, in which case the bars are almost unaffected. The growth of a $0.05 \% M_{\star}$ $\mathrm{BH}$ breaks the dynamical equilibrium of the two decoupled bars within a short time. The relative position angle of the two bars, $\Delta \phi$, for all of these models is shown in the inset of Figure 2. Although $A_{\text {in }}$ decreases sharply because of the growth of $\mathrm{BH}$, the pattern speed does not change much during the inner bar weakening, e.g., $t=300-420$ in the case of $M_{\mathrm{bh}} / M_{\star}=$ $0.05 \%$. As the inner bar still rotates independently of its outer counterpart, they are still interacting strongly. The evolution of $\Delta \phi$ shows that the weakened inner bars may be trapped by the outer bars, i.e., coupling to alignment. The coupling process is essentially similar to the case of the aligned S2B presented in Du et al. (2015, Figure 11): the fast-rotating inner bar slows down sharply before reaching a perpendicular orientation, falling into alignment with the outer bar. The key difference from the aligned S2B of that earlier model is that the coupling leaves no prominent face-on peanut-shaped density contours. The amplitude of the aligned "inner" bar decreases slowly in the later stage, until it becomes quite round, probably indicating continued orbital scattering. In order to verify that the evolution found here is not an artifact of the grids and code use, we rerun the simulations of bar dissolution $(t=300-400)$ with the momentum-conserving treecode gyrfalcON (Dehnen 2000, 2014). ${ }^{9}$ Adaptive time steps are used to accurately integrate particle motions close to the BHs. Figure 2 includes the results from these tests; in all cases, the results are consistent with those from GALAXY.

Because many massive BHs are already present in the very early universe, likely before disks and bars were established (Fan et al. 2001, 2003), we also model this scenario by adding a $\mathrm{BH}$ of fixed mass from the beginning of the simulations. In order to generate the initial disk for the S2B instability, we use the same initial conditions as the standard S2B model. Because

\footnotetext{
9 Available at the NEMO repository (Teuben 1995): http://admit.astro.umd. edu/nemo/.
} 

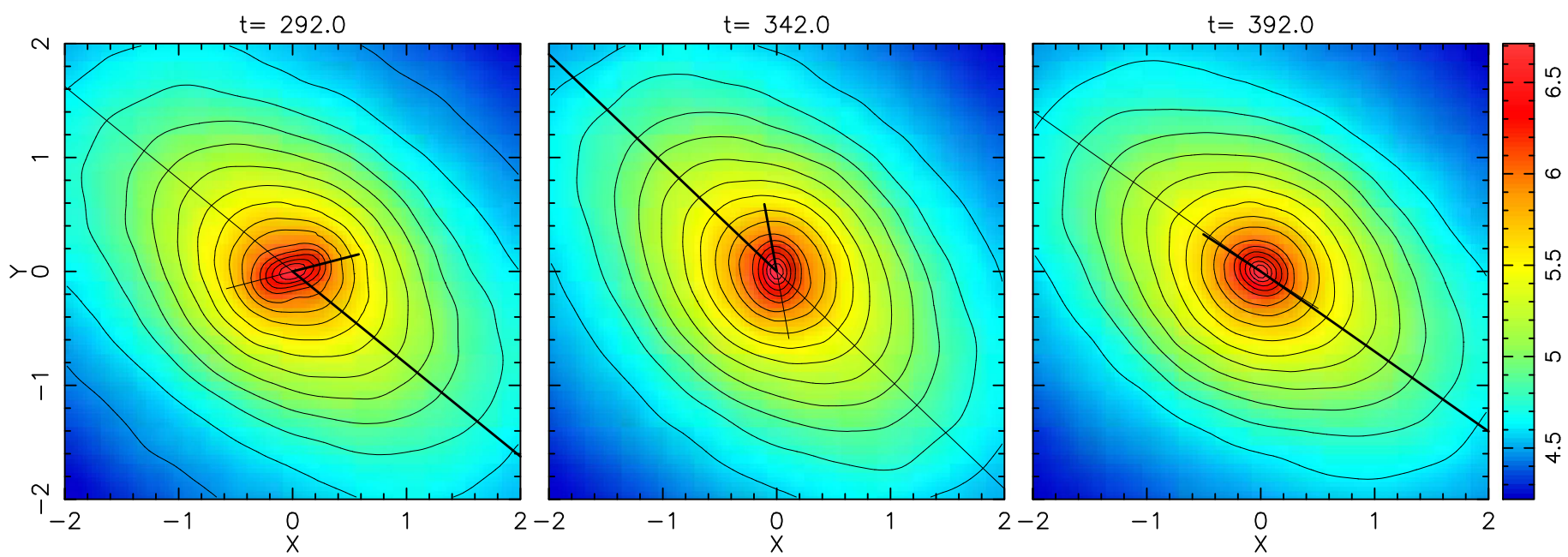

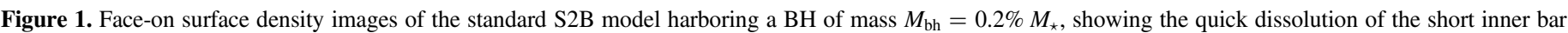

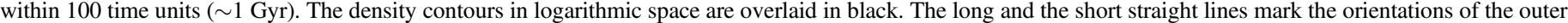
and the inner bars, respectively.

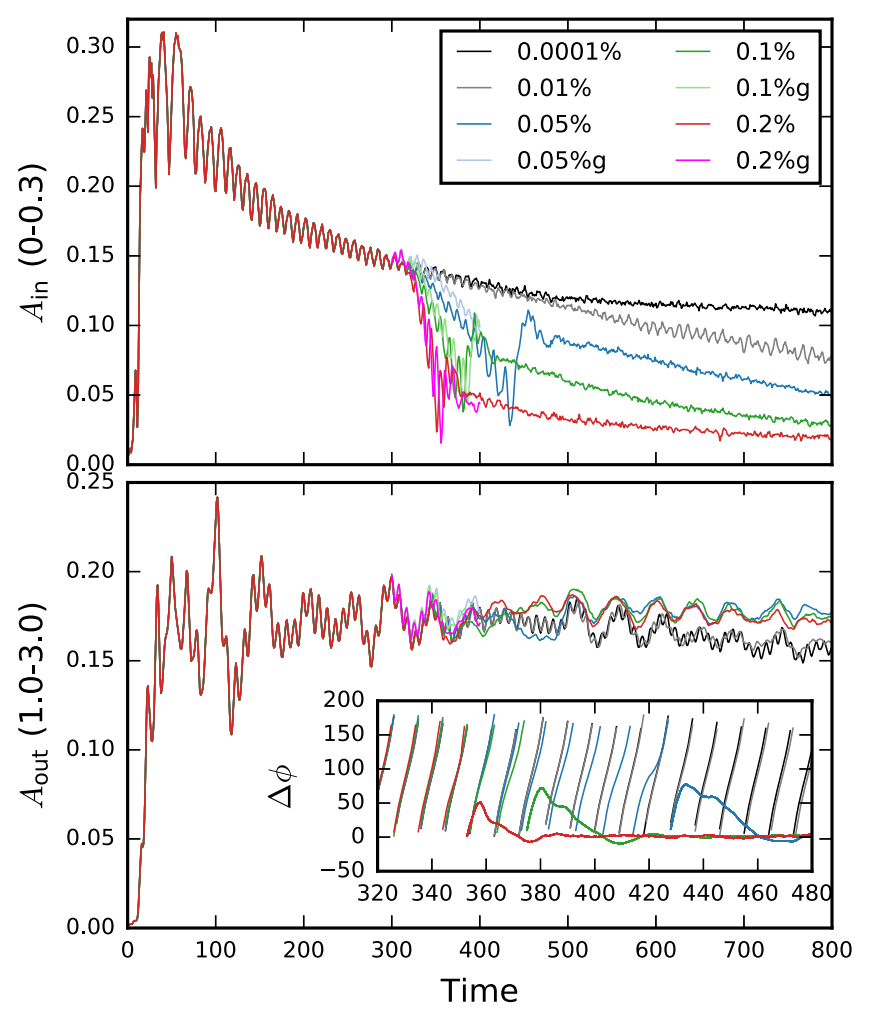

Figure 2. Time evolution of the inner (top) and the outer (bottom) bars of the standard S2B model under the dynamical influence of BHs. The BHs grow to the maximum mass $M_{\mathrm{bh}}$ (shown in the legend) from a seed of mass $0.0001 \% M_{\star}$ during $t=300-350$. The cases of $M_{\mathrm{bh}} / M_{\star}=0.0001 \%-0.2 \%$ are shown here. $A_{\text {in }}$ and $A_{\text {out }}$ are defined as the Fourier $m=2$ amplitudes averaged over $R \leqslant 0.3$ and $1.0 \leqslant R \leqslant 3.0$, respectively. The relative position angle of the two bars, $\Delta \phi$, as a function of time is shown in the inset. For cross-check, the results of $M_{\mathrm{bh}} / M_{\star}=0.05 \%-0.2 \%$ recalculated using gyrfalcON are labeled as $0.05 \% \mathrm{~g}, 0.1 \% \mathrm{~g}$, and $0.2 \% \mathrm{~g}$, respectively.

of the chaotic nature of galactic disks (Sellwood \& Debattista 2009), stochasticity can significantly affect such models (Du et al. 2015). Therefore, we run 10 S2B simulations for each BH mass changing only the random seed for generating the initial particles. In the case of a pre-existing $\mathrm{BH}$, within $\sim 1 \mathrm{Gyr}$, the initial disk generally generates an $\mathrm{S} 2 \mathrm{~B}$ structure similar to that in the standard S2B model. With a pre-existing BH of mass $0.2 \% M_{\star}$, we never obtain a steady S2B feature lasting more than $1.5 \mathrm{Gyr}$. In the case of a $0.05 \% M_{\star} \mathrm{BH}$, half the simulations maintain decoupled $\mathrm{S} 2 \mathrm{~B}$ features for more than 4 Gyr.

\subsection{Other Models}

In the clumpy S2B model, the strong inner bar extends to $1.5 \mathrm{kpc}$, while the outer bar is quite weak. Thus, the gravitational torque of the outer bar has a negligible effect on the robustness of the inner bar. We regard this model as an extreme case for testing the robustness of short bars under the dynamical influence of $\mathrm{BH}$ growth. The inner bar in this case can be destroyed within 2 Gyr by a $\mathrm{BH}$ of mass $0.3 \% M_{\star}$ without coupling between the two bars. We have examined that a single nuclear bar performs similarly to the inner bar of the clumpy S2B model. Using a single-barred simulation ( $a_{\mathrm{Bar}} \sim 3-4 \mathrm{kpc}$ ), Valluri et al. (2016) investigated the change of the orbital families that is induced by the growth of BHs. They showed that BHs of mass $0.2 \% M_{\star}$ can destroy most barsupporting orbits within $R<1.5 \mathrm{kpc}$, which is consistent with our result. Therefore, $0.2 \% M_{\star}$ is likely to be the maximum $\mathrm{BH}$ mass allowing the presence of short inner bars in galaxies free of mergers.

\section{The $M_{b h}-M_{\star}$ Relation}

If short bars are the primary drivers of gas inflows feeding BHs, the dissolution of short bars should halt the secular growth of BHs. Thus, the mass of BHs may stall at $\sim 0.1 \% M_{\star}$. In observations, $M_{\star}$ is generally approximated by assuming reasonable mass-to-light $\left(M_{\star} / L\right)$ ratios. Many short bars may have been destroyed after they played an important role in feeding BHs. After short bars are destroyed, continuing star formation in spiral galaxies will result in an even lower mass ratio of BH-to-host galaxy. Our simulations therefore constrain the maximum $M_{\mathrm{bh}} / M_{\star}$ allowed by secular evolution.

In Figures 3 and 4, we show different measurements of $M_{\mathrm{bh}} / M_{\star}$ from Kormendy \& Ho (2013) and Reines \& Volonteri (2015), respectively. The criterion obtained from the simulations is overlaid as the shaded region. In Figure 3, except for 


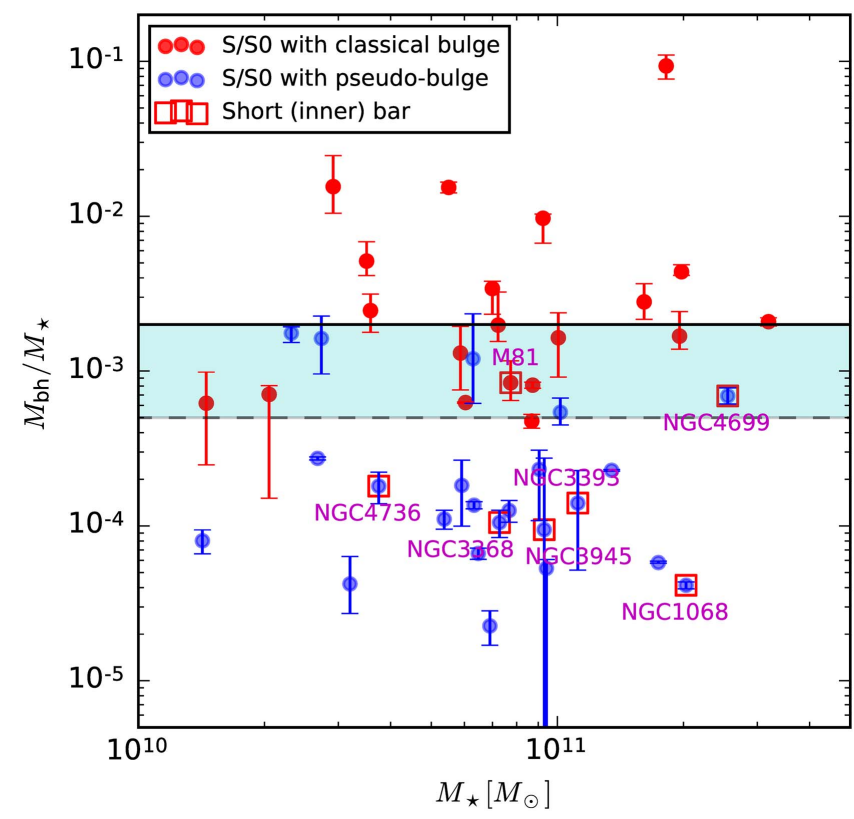

Figure 3. Mass ratios of BH-to-host-galaxy stellar mass summarized in Kormendy \& Ho (2013). The BH mass and stellar mass of NGC4699 are adopted from Saglia et al. (2016) and Erwin et al. (2015), respectively. The red and blue dots represent the galaxies with classical bulges and pseudo-bulges, respectively. Their $\mathrm{BH}$ masses are all measured via stellar dynamics. The error comes from the uncertainty in $M_{\mathrm{bh}}$ measurement. The maximum $\mathrm{BH}$ mass fraction obtained from our simulations is overlaid in the cyan shaded region. The galaxies having a short (inner) bar are marked with red squares.

NGC4699, all the data are taken from Kormendy \& Ho (2013), where the $\mathrm{BH}$ masses are measured using dynamical methods and the stellar masses are based on the $M_{\star} / L$ of Bell et al. (2003). These spiral/S0 galaxies are classified into two groups based on the morphology of their bulges. According to the decomposition of Erwin et al. (2015), NGC4699 is a galaxy with a composite bulge containing a primary pseudo-bulge (mass $\sim 36 \% M_{\star}$ ) and a small classical bulge $\left(\sim 11 \% M_{\star}\right)$. Thus, NGC4699 should also be classified as a galaxy with a pseudo-bulge. Disk galaxies with pseudo-bulges tend to fall below the mass ratio of $\mathrm{BH}$-to-host galaxy found in galaxies with classical bulges and elliptical galaxies (Reines \& Volonteri 2015). Such a clear separation suggests that the BHs evolve differently. Exponential, disky pseudo-bulges are generally expected to be generated by internal secular evolution without significant mergers. Therefore, the growth of BHs in galaxies with pseudo-bulges and bulgeless galaxies should be dominated by secular processes, including inner-bar-driven gas inflows.

Mergers are rare in the local universe. Most active galactic nuclei (AGNs) may also be triggered by secular processes. Thus, the $\mathrm{BH}$ masses in AGN host galaxies are likely to be lower than the maximum $\mathrm{BH}$ mass allowed by secular evolution. In Figure 4, we include the measurements of 256 broad-line AGNs from Reines \& Volonteri (2015) and 15 reverberation-mapped AGNs from Bentz \& Katz (2015). The stellar masses in Figure 3 are systematically higher by a factor of $\sim 2$ than those in Figure 4, which were estimated by the color-dependent $M_{\star} / L$ presented by Zibetti et al. (2009). The histogram in the right panel of Figure 4 shows the number distribution of the mass ratio of the AGN host galaxies and the galaxies with pseudo-bulges. Less galaxies are located at the cyan shaded region; mass ratios larger than 0.002 are rare.
Thus, maximum BH mass suggested by our simulations is roughly consistent with the observations. It suggests that $\mathrm{BH}$ growth via secular evolution is terminated by the dissolution of short bars.

Short inner bars are expected to be gradually destroyed once $M_{\mathrm{bh}} \sim 0.05 \%-0.2 \% M_{\star}$. Five S2Bs (NGC1068, NGC3368, NGC3393, NGC3945, and NGC4736; see Erwin 2004) are marked by red squares in Figures 3 and 4. All of them have lower mass ratios than the upper boundary of the cyan region. In M81, a short inner bar may be embedded in a weak outer bar (Gutiérrez et al. 2011), although it is generally considered to have a significant classical bulge. NGC4699 only harbors a single short bar. The absence of strong outer bars allows short bars to persist for longer because of the lack of interaction between two bars. Thus, it is not surprising that M81 and NGC4699 are present at the cyan shaded region. However, no $\mathrm{S} 2 \mathrm{Bs}$ are above this range.

\section{Conclusions}

$\mathrm{BH}$ growth in disk galaxies without significant mergers is expected to be driven by secular processes. Sub-kiloparsecscale short bars, generally embedded in large-scale bars, have been hypothesized to be an important mechanism for driving gas inflows, feeding BHs. Whether and under what conditions short bars can survive the presence of a $\mathrm{BH}$ can provide a crucial test of this scenario. By adding BHs at the center of short-bar simulations, we have found that short bars are likely to be destroyed by BHs with masses at least $0.05 \%-0.2 \%$ of the galaxy stellar mass; mass ratios larger than $0.2 \% M_{\star}$ always lead to destruction of short bars. This maximum mass ratio coincides well with the observed upper limit on $\mathrm{BH}$ mass ratios for real disk galaxies. Therefore, we provide a possible explanation for the lower $M_{\mathrm{bh}} / M_{\star}$ in disk galaxies: the $\mathrm{BH}$ growth via secular evolution may be terminated by the dissolution of short inner bars.

We sketch a potential evolutionary path of the central structures of disk galaxies as follows. In gas-rich galaxies, gas is funneled into central regions by large-scale galactic bars, gradually building a stellar nuclear disk. Short inner bars form spontaneously in nuclear disks after sufficient accumulation of dynamically cold stellar material (Du et al. 2015). Then, short inner bars funnel gas further into the center, feeding BHs. According to the criterion in this Letter, inner bars are destroyed when the $\mathrm{BH}$ mass grows to $\sim 0.1 \% M_{\star}$. The dissolution of inner bars in turn slows down, or even stops, the growth of BHs. Thus, BH growth in disk galaxies is mediated by the secular evolution of short bars in the absence of mergers.

However, previous observations have not found a clear relation between inner bars and AGNs (e.g., Regan \& Mulchaey 1999; Martini et al. 2001; Erwin \& Sparke 2002; Laine et al. 2002). One possible reason is that catching $\mathrm{BH}$ fueling in the act is challenging, as one AGN activity episode typically lasts just a few million years. Another possible reason is that AGN fueling may be dominated by some unknown stochastic processes very local to the $\mathrm{BH}$, as the radius that inner-bar-driven gas inflows reach may still be too far away from the BH's accretion disk. Or the fraction of short inner bars may be underestimated because of dust obscuration, particularly in late-type galaxies. Furthermore, other non-axisymmetric nuclear structures, e.g., spirals, may also drive gas inflows feeding BHs (Hopkins et al. 2010). Thus, AGNs may not be completely stalled after inner bars are destroyed. Therefore, it is 


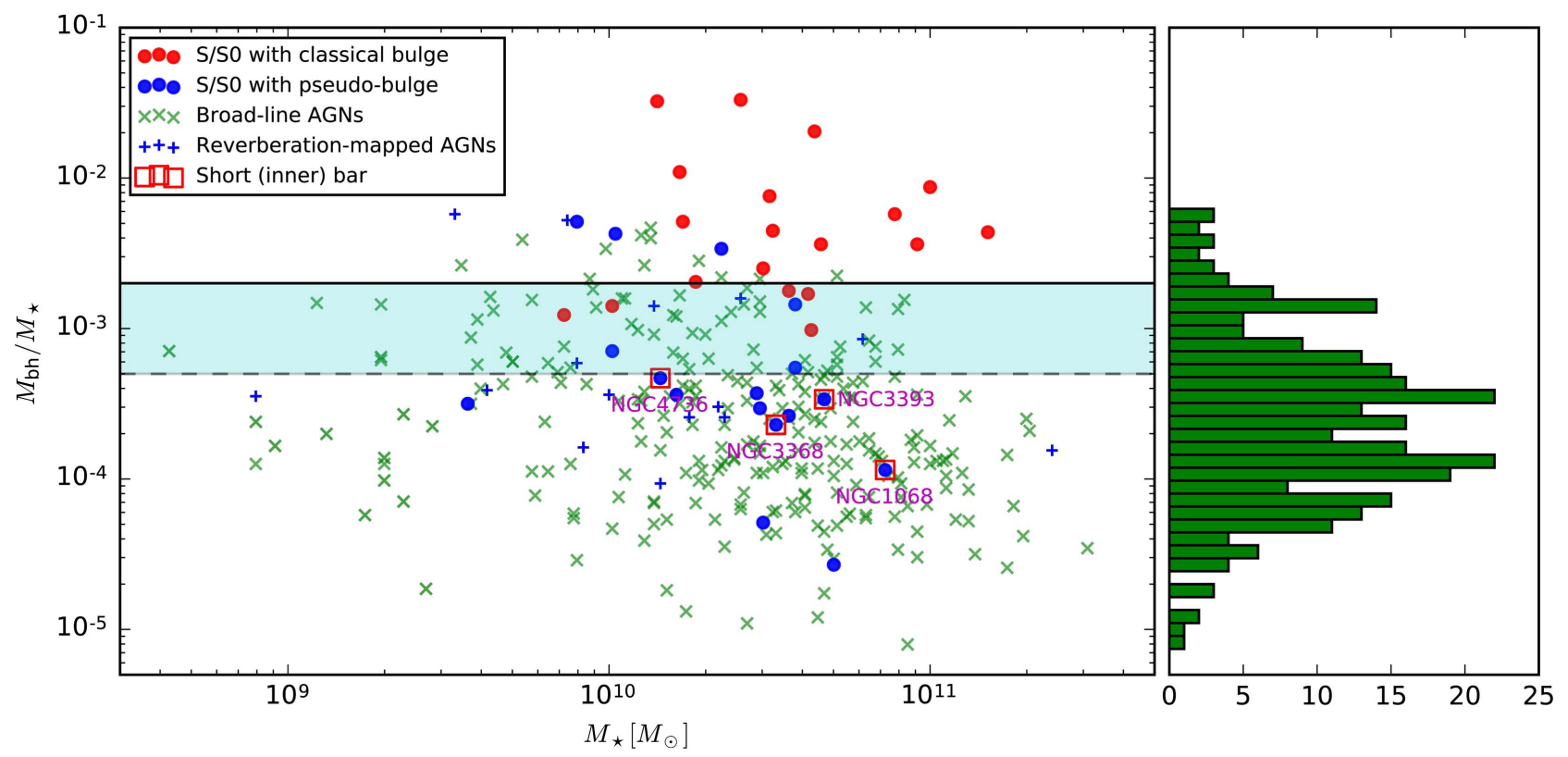

Figure 4. Mass ratios of BH-to-host-galaxy stellar mass estimated by Reines \& Volonteri (2015). The BH masses of 256 broad-line AGNs (green crosses, including 12 dwarf galaxies) and 15 reverberation-mapped AGNs (blue plusses) are adopted from Reines \& Volonteri (2015) and Bentz \& Katz (2015), respectively. The blue and red dots represent the objects from Kormendy \& Ho (2013) shown above in Figure 3, BH masses for which are measured by stellar dynamics. Stellar masses are estimated using the same method as the AGN host galaxies to avoid systematic deviation. The red squares mark the galaxies having short (inner) bars. NGC3945 and NGC4699 are not included in Reines \& Volonteri (2015). In the right panel, the histogram shows the number distribution of mass ratios of the AGN host galaxies and galaxies with pseudo-bulges.

perhaps not surprising that no clear relation is reported. The previous studies cannot rule out short bars as a potentially important mechanism of promoting BH growth in disk galaxies. Whether or not other drivers of gas inflows are also suppressed by $\mathrm{BH}$ growth will be studied in future work.

M.D. thanks the Jeremiah Horrocks Institute of the University of Central Lancashire for their hospitality during a three-month visit while this Letter was in progress. We acknowledge support from an Newton Advanced Fellowship awarded by the Royal Society and the Newton Fund, and from the CAS/SAFEA International Partnership Program for Creative Research Teams. The research presented here is partially supported by the 973 Program of China under grant No. 2014CB845700, by the National Natural Science Foundation of China under grant Nos. 11333003, 11322326, and by a China-Chile joint grant from CASSACA. This work made use of the facilities of the Center for High Performance Computing at Shanghai Astronomical Observatory. V.P.D. is supported by STFC Consolidated grant ST/M000877/1 and acknowledges the personal support of George Lake, and of the Pauli Center for Theoretical Studies, which is supported by the Swiss National Science Foundation (SNF), the University of Zürich, and ETH Zürich. The work of L.C.H. was supported by National Key Program for Science and Technology Research and Development grant 2016YFA0400702.

\section{References}

Athanassoula, E., Lambert, J. C., \& Dehnen, W. 2005, MNRAS, 363, 496 Bell, E. F., McIntosh, D. H., Katz, N., \& Weinberg, M. D. 2003, ApJS, 149,289

Bentz, M. C., \& Katz, S. 2015, PASP, 127, 67

Cisternas, M., Jahnke, K., Inskip, K. J., et al. 2011, ApJ, 726, 57
Croton, D. J., Springel, V., White, S. D. M., et al. 2006, MNRAS, 365, 11 Dehnen, W. 2000, ApJL, 536, L39

Dehnen, W. 2014, gyrfalcON: N-body code, Astrophysics Source Code Library, ascl:1402.031

Di Matteo, T., Springel, V., \& Hernquist, L. 2005, Natur, 433, 604

Dong, X.-B., Ho, L. C., Yuan, W., et al. 2012, ApJ, 755, 167

Du, M., Debattista, V. P., Shen, J., \& Cappellari, M. 2016, ApJ, 828, 14

Du, M., Shen, J., \& Debattista, V. P. 2015, ApJ, 804, 139

Erwin, P. 2004, A\&A, 415, 941

Erwin, P. 2011, MSAIS, 18, 145

Erwin, P., Saglia, R. P., Fabricius, M., et al. 2015, MNRAS, 446, 4039

Erwin, P., \& Sparke, L. S. 2002, AJ, 124, 65

Eskridge, P. B., Frogel, J. A., Pogge, R. W., et al. 2000, AJ, 119, 536

Fan, L., Fang, G., Chen, Y., et al. 2014, ApJL, 784, L9

Fan, X., Narayanan, V. K., Lupton, R. H., et al. 2001, AJ, 122, 2833

Fan, X., Strauss, M. A., Schneider, D. P., et al. 2003, AJ, 125, 1649

Filippenko, A. V., \& Ho, L. C. 2003, ApJL, 588, L13

Gabor, J. M., Impey, C. D., Jahnke, K., et al. 2009, ApJ, 691, 705

Georgakakis, A., Coil, A. L., Laird, E. S., et al. 2009, MNRAS, 397, 623

Gerhard, O. E., \& Binney, J. 1985, MNRAS, 216, 467

Greene, J. E., Peng, C. Y., Kim, M., et al. 2010, ApJ, 721, 26

Greene, J. E., Seth, A., Kim, M., et al. 2016, ApJL, 826, L32

Gutiérrez, L., Erwin, P., Aladro, R., \& Beckman, J. E. 2011, AJ, 142, 145

Hopkins, P. F., Bundy, K., Croton, D., et al. 2010, ApJ, 715, 202

Hopkins, P. F., \& Quataert, E. 2010, MNRAS, 407, 1529

Hozumi, S. 2012, PASJ, 64, 5

Jiang, N., Ho, L. C., Dong, X.-B., Yang, H., \& Wang, J. 2013, ApJ, 770, 3

Jiang, Y.-F., Greene, J. E., Ho, L. C., Xiao, T., \& Barth, A. J. 2011, ApJ, 742, 68

Kocevski, D. D., Faber, S. M., Mozena, M., et al. 2012, ApJ, 744, 148

Kormendy, J., \& Ho, L. C. 2013, ARA\&A, 51, 511

Laine, S., Shlosman, I., Knapen, J. H., \& Peletier, R. F. 2002, ApJ, 567, 97

Martini, P., Pogge, R. W., Ravindranath, S., \& An, J. H. 2001, ApJ, 562, 139

Menéndez-Delmestre, K., Sheth, K., Schinnerer, E., Jarrett, T. H., \& Scoville, N. Z. 2007, ApJ, 657, 790

Regan, M. W., \& Mulchaey, J. S. 1999, AJ, 117, 2676

Reines, A. E., \& Volonteri, M. 2015, ApJ, 813, 82

Saglia, R. P., Opitsch, M., Erwin, P., et al. 2016, ApJ, 818, 47

Schawinski, K., Treister, E., Urry, C. M., et al. 2011, ApJL, 727, L31

Sellwood, J. A. 2014, arXiv:1406.6606

Sellwood, J. A., \& Debattista, V. P. 2009, MNRAS, 398, 1279

Shen, J., \& Sellwood, J. A. 2004, ApJ, 604, 614 
Shlosman, I., Frank, J., \& Begelman, M. C. 1989, Natur, 338, 45

Simmons, B. D., Lintott, C., Schawinski, K., et al. 2013, MNRAS, 429, 2199

Teuben, P. 1995, in ASP Conf. Ser. 77, Astronomical Data Analysis Software and Systems IV, ed. R. A. Shaw, H. E. Payne, \& J. J. E. Hayes (San Francisco CA: ASP), 398
Toomre, A. 1977, in Evolution of Galaxies and Stellar Populations, ed. B. M. Tinsley, R. B. G. Larson, \& D. Campbell (New Haven, CT: Yale Univ. Observatory), 401

Valluri, M., Shen, J., Abbott, C., \& Debattista, V. P. 2016, ApJ, 818, 141

Zibetti, S., Charlot, S., \& Rix, H.-W. 2009, MNRAS, 400, 1181 